\title{
Sarcoma indiferenciado (embrionario) de hígado en el adulto*
}

\author{
Drs. EDUARDO CODDOU C..$^{1,2}$, JAVIER LÓPEZ S..$^{1,2}$, \\ JUAN MADARIAGA G. ${ }^{1,3}$, MARCELO BARRA M..$^{1,2}$ \\ - Servicio de Cirugía. Hospital de Coquimbo. \\ Instituto de Cirugía. Universidad Católica del Norte. \\ 3 Servicio de Anatomía Patológica. Hospital de Coquimbo. \\ Coquimbo, Chile.
}

\begin{abstract}
Undifferentiated (Embryonal) sarcoma of the liver in adults

We report a 48 years old women presenting with a painful abdominal mass and hepatomegaly. An abdominal CAT scan showed a focal lesion of $14 \mathrm{~cm}$ diameter in liver segment 4 . The patient was subjected to a left hepatectomy with a normal postoperative evolution. The pathological diagnosis of the surgical piece was an undifferentiated sarcoma. Nine months later, a local relapse was detected and the patient died 22 months after the operation.
\end{abstract}

Key words: Undifferentiated sarcoma, hepatic tumors, surgery, chemotherapy.

\section{Resumen}

Introducción: Los sarcomas indiferenciados (embrionario) del hígado (SIEH) son neoplasias infrecuentes. Se presentan principalmente en edad pediátrica y son considerados de mal pronóstico. En adultos, existen 71 pacientes publicados en el mundo, y en nuestro país no hay casos descritos en este grupo etario. Objetivos: Presentar el caso de una paciente adulta portadora de un SIEH, que fue sometida a cirugía resectiva y realizar una revisión del tema. Caso clínico: Mujer de 48 años de edad, con historia de dolor abdominal, masa palpable en epigastrio y hepatomegalia. Marcadores tumorales negativos, tomografía abdominal revela lesión focal de 14 centímetros en segmento 4 . Se aborda quirúrgicamente, biopsia rápida revela tumor sólido maligno indiferenciado, y se realiza hepatectomía izquierda. Evolución postoperatoria favorable. Mediante el análisis histopatológico, histoquímico e inmunohistoquímico se diagnostica un SIEH y se corrobora una resección R0. Al noveno mes de evolución se pesquisa foco de recidiva hepática, inicia progresivo compromiso del estado general, falleciendo 22 meses después de la cirugía. Discusión: Actualmente se recomienda resección completa del tumor seguido de quimioterapia coadyuvante, con lo cual se han logrado sobrevidas libres de enfermedad mayores a 5 años. El dar a conocer las experiencias de casos aislados en esta patología tan infrecuente, permitiría aumentar la casuística mundial, mejorar las técnicas de enfrentamiento, y evaluar el impacto de la quimioterapia en el pronóstico.

Palabras clave: Sarcoma indiferenciado, tumores hepáticos, cirugía, quimioterapia.

*Recibido el 3 de Abril de 2010 y aceptado para publicación el 27 de Abril de 2010.

Correspondencia: Dr. Eduardo Coddou C.

Casilla 207, Coquimbo, Chile.

E-mail: ecoddou@gmail.com 


\section{Introducción}

Los sarcomas primarios del hígado son lesiones infrecuentes y representan menos del $1 \%$ de los tumores hepáticos primarios ${ }^{1-3}$. Aquí se incluye un grupo heterogéneo de distintos tipos histológicos como el angiosarcoma, leiomiosarcoma, fibrosarcoma, hemangioendotelioma epiteloide, histiocitoma fibroso maligno, y el sarcoma indiferenciado (embrionario) del hígado (SIEH). Este último se presenta con más frecuencia en pacientes pediátricos durante la primera década de vida y es considerado una neoplasia agresiva con un pronóstico desfavorable ${ }^{1-16}$. Inicialmente fue descrito como mesenquimoma por Donovan y Santull, posteriormente Scout acuña el término mesenquimoma maligno y a partir de sus entidades clinicopatológicas, Stocker e Ishak en 1978 lo denominan como SIEH ${ }^{4-7}$.

Existen reportes de pacientes sobre quince años de edad, del año 2007 y 2008 que describen sólo 71 casos de SIEH en adultos publicados en el mun$\mathrm{do}^{1,4,8,9}$. Debido a su baja incidencia, no existe la experiencia para llegar a un consenso en su manejo; siendo la cirugía, con o sin quimioterapia coadyuvante el tratamiento más aceptado.

Presentamos el caso de una paciente adulta portadora de SIEH sometida a cirugía, y realizamos una revisión del tema.

\section{Caso clínico}

Mujer de 48 años de edad, con antecedentes de hipertensión arterial, colecistectomía a los 30 años y ooferectomía izquierda a los 39 años. Ingresa a nuestro servicio, derivada desde otro hospital, por un cuadro de cuatro días de evolución, caracterizado por intenso dolor epigástrico de tipo urente e inicio súbito, asociado a escasos vómitos. No refiere sintomatología previa. $\mathrm{Al}$ examen físico destacaba una paciente en buenas condiciones generales, hemodinámicamente estable, afebril, con dolor intenso en relación a masa palpable en epigastrio, hipocondrio derecho e hígado, que se palpaba $3 \mathrm{~cm}$ bajo el reborde costal derecho. Requirió de hospitalización y manejo del dolor con opiáceos. Se solicita ecografía abdominal, que reveló masa hepática de $10 \times 9 \times 8$ $\mathrm{cm}$ en relación al lóbulo hepático izquierdo. En los estudios de laboratorio destacaba una función hepática y renal normal, bilirrubinemia y transaminasas normales, y sólo leve elevación de las fosfatasas alcalinas. Marcadores tumorales como $\alpha$-fetoproteína $\mathrm{y}$ antígeno carcinoembrionario negativos, al igual que la serología para hepatitis B y C. Tomografía computada de abdomen (TC) revela lesión focal de $14 \mathrm{~cm}$ de diámetro ubicada en el segmento 4 , de contornos bien delimitados y densidad heterogénea con áreas líquidas y componente hiperdenso (Figura 1). La lesión abarcaba desde la cúpula hepática, alcanzando en profundidad hasta las venas hepáticas izquierda y media, a escasos centímetros de la vena cava superior y desplazando la vena porta hacia posterior.

Se aborda quirúrgicamente mediante laparotomía subcostal bilateral, biopsia rápida informa tumor sólido maligno indiferenciado, y se realiza hepatectomía izquierda (Figura 2). Paciente evoluciona sin complicaciones, dándose de alta al octavo día postoperatorio. Vuelve a su domicilio, alejado de nuestro centro, perdiéndose de los controles indicados. En anatomía patológica se recibe lóbulo hepático izquierdo de 840 gr, de 19 × 13 × $8 \mathrm{~cm}$. Al corte se reconoce lesión tumoral no encapsulada, con focos hemorrágicos y extensa necrosis central, de crecimiento expansivo, de 14 × 9 × 5 $\mathrm{cm}$. El estudio microscópico muestra una proliferación de células pleomórficas y fusocelulares, con núcleos hipercromáticos, dispuestas en un estroma colagenoso. Además, se reconocen algunas células multinucleadas. La lesión no alcanza los bordes de sección quirúrgicos y dista $1,5 \mathrm{~cm}$ de éste. Con la tinción de PAS y PAS diastasa se reconocen glóbulos hialinos intra y extracitoplasmáticos. El estudio inmunohistoquímico con anticuerpos monoclonales para alfa-1-antitripsina fue positivo en los glóbulos eosinófilos y negativo para citoqueratinas totales (AE1/AE3), Hepar-1 y Vimentina. Se concluye que los hallazgos histopatológicos son compatibles con SIEH. (Figuras 3 y 4 ).

Nueve meses después de la cirugía consulta por dolor en hipocondrio derecho. La TC revela nódulo hepático sólido, planteándose un foco de recidiva (Figura 5). Fallece veintidós meses después de su cirugía.

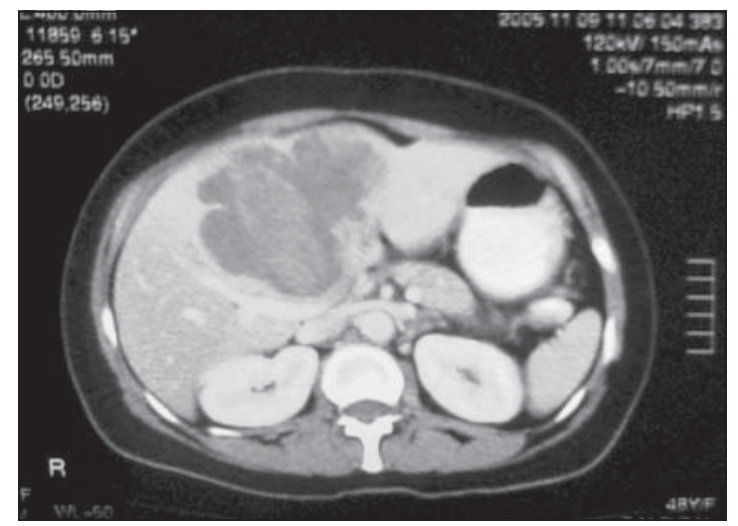

Figura 1. TC que revela la lesión primaria. 


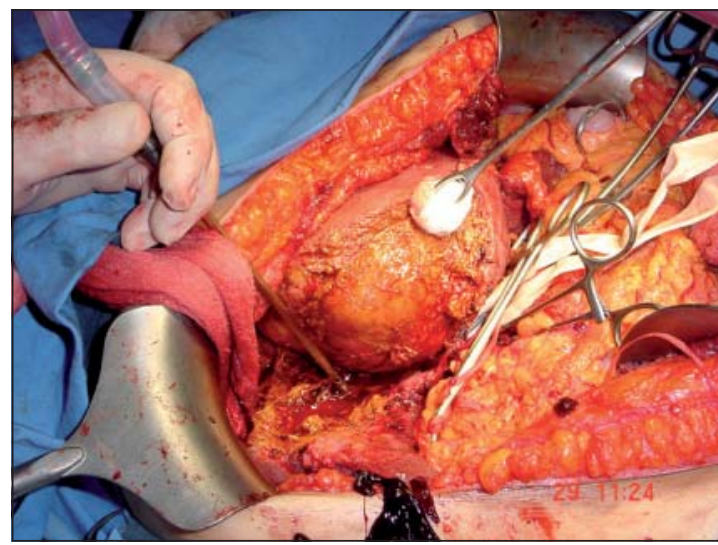

Figura 2. Tumor intraoperatorio.

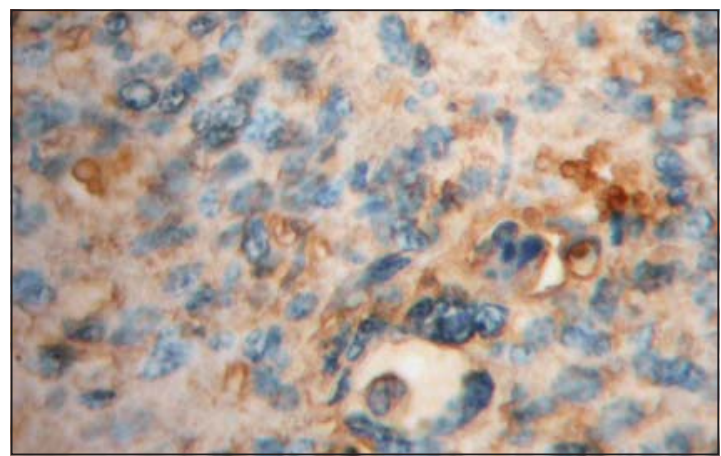

Figura 4. Alfa-1-antitripsina 40x. positiva en los glóbulos intra y extracitoplasmáticos.

\section{Discusión}

La edad media de presentación del SIEH en adultos es de 25 años. Sobre los 48 años se han publicado sólo quince pacientes ${ }^{4}$. La relación hombre/mujer es de 1:1,3. Su presentación clínica es inespecífica, similar a la gran mayoría de los tumores hepáticos primarios; con dolor abdominal alto, con o sin masa palpable. Puede o no acompañarse de síntomas sistémicos, fiebre (asociado a necrosis tumoral), baja de peso y alteración del tránsito intestinal. Existen distintas formas de presentación, muchas de ellas en forma aguda, como la de nuestro caso; se describe un paciente que simulaba una apendicitis aguda ${ }^{10}$, producto de un hemoperitoneo con irritación peritoneal en fosa ilíaca derecha, secundario a ruptura hepática espontánea por SIEH.

Imagenológicamente pueden presentar un amplio espectro de componentes sólidos o líquidos. Un estudio que recolectó 28 pacientes entre 3 y 49 años

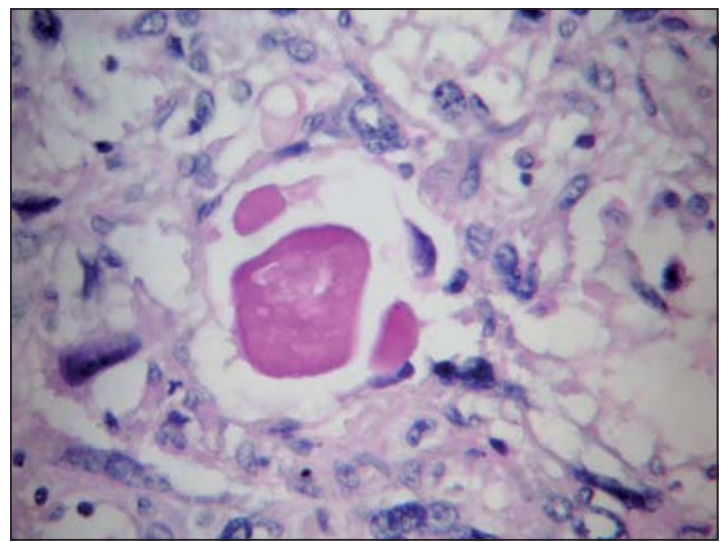

Figura 3. Tinción Pas 40x glóbulos hialinos PAS positivos y en la periferia algunas células pleomórficas.

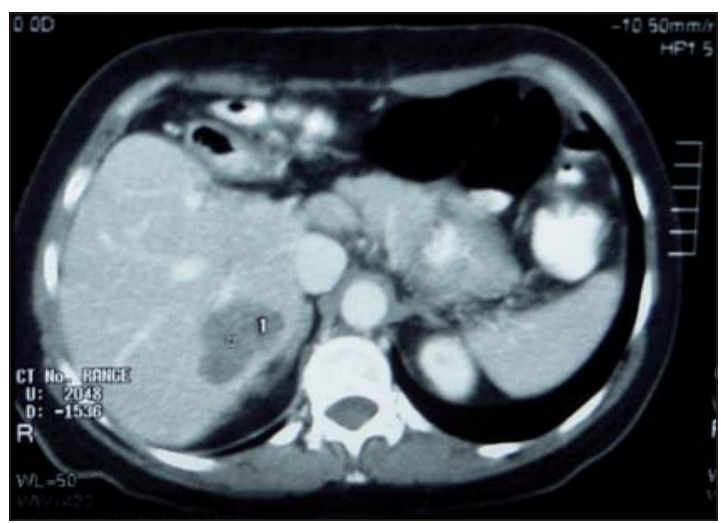

Figura 5. TC que revela la recidiva.

describe que el diámetro promedio del tumor fue de $14 \mathrm{~cm}$ (márgenes entre 10 y $25 \mathrm{~cm})^{5}$. Todos los tumores son predominantemente sólidos $(83 \%$ como promedio por lesión), con componentes mixoideo, hemorrágico y gelatinoso. En la ultrasonografía los tumores aparecen principalmente de aspecto sólidos con componente iso o hiperecogénicos. Áreas de aspecto quístico aparecen en un promedio del 19\% del volumen tumoral. Dentro de los hallazgos tomográficos todos los tumores demostraban una densidad líquida, y áreas de densidad intermedia fueron notados en su periferia. En cinco casos se determinó zonas centrales con incremento de la atenuación, compatible con hemorragias agudas. En la resonancia magnética estos tumores mostraron señales de alta intensidad similar al líquido cefalorraquídeo en un $89 \%$ del volumen tumoral.

Por sus características y baja frecuencia, es poco probable que se plantee el diagnóstico de SIEH en el preoperatorio. Entre las lesiones solitarias predo- 
minantemente sólidas, con degeneración quística parcial, existen muchas posibilidades diagnósticas, como: carcinoma hepatocelular, colangiocarcinoma intrahepático, hemangioendotelioma epiteloide, linfomas, angiosarcoma, otros sarcomas, metástasis y adenoma hepatocelular. También en un menor número de casos, estas lesiones se podrían presentar con un mayor porcentaje de componente quístico, lo cual haría plantear otras neoplasias como cistoadenoma biliar, cistoadenocarcinoma, degeneración quística de carcinoma hepatocelular o de metástasis; también sería planteable algunas lesiones benignas como abscesos hepáticos (bacterianos o parasitarios) o evolución de un hematoma postraumático.

Frente a estos cuadros clínicos en donde las posibilidades diagnósticas de un hepatocarcinoma, metástasis o cuadro infeccioso hayan disminuido o descartado, recomendamos el abordaje quirúrgico, siempre y cuando el estudio preoperatorio establezca que se trate de una lesión solitaria, resecable y un paciente apto para soportar una cirugía de esta envergadura. La biopsia percutánea estaría recomendada en pacientes con contraindicación para una resección formal, en quienes se podría optar por terapias sistémicas o de embolización segmentaria. $\mathrm{La}$ biopsia intraoperatoria para estos tumores es poco concluyente, sin embargo, nos puede orientar y/o confirmar que estamos frente a una lesión neoplásica indiferenciada, que si se corrobora con el estudio preoperatorio y los hallazgos intraoperatorios de estar localizado sería subsidiaria de una resección hepática de tipo oncológico, como fue el caso que presentamos.

La histogénesis de estos tumores es controversial. Histopatológicamente se describen como principales características el crecimiento expansivo, áreas hemorrágicas y extensa necrosis ${ }^{8,11}$. Las células son de tamaño mediano a grande, fusocelulares, con marcado pleomorfismo o multinucleadas. Además, se reconocen gránulos intra y extracelulares, PAS positivos y PAS diastasa resistentes. El estudio inmunohistoquímico no tiene un patrón específico, pero es útil en excluir otros tumores.

Al SIEH se le atribuía un pésimo pronóstico, sin embargo, hoy se han agregado nuevas experiencias en su manejo, empleando tratamientos con los cuales se han logrado sobrevidas libres de enfermedad por sobre los cinco años ${ }^{1}$. Lo primordial en el tratamiento del SIEH es lograr una cirugía con resección completa $\mathrm{R} 0^{1,2,4,12}$. Existe poca evidencia con respecto a la quimioterapia coadyuvante; por los escasos pacientes publicados, sin embargo, existe una tendencia objetivada en estas publicaciones, que estos tumores serían sensibles a la quimioterapia, mejorando la sobrevida en los pacientes sometidos a cirugía $\mathrm{R} 0^{1-3,12-14}$.
Lenze y colaboradores ${ }^{1}$ revisaron los casos publicados, y detectaron 48 pacientes con seguimiento completo. La sobrevida actuarial para uno y dos años fue de $61 \%$ y $55 \%$ respectivamente, y la sobrevida media fue de 29 meses. Al comparar los pacientes sometidos a cirugía R0 más quimioterapia coadyuvante con aquellos que sólo fueron sometidos a cirugía R0 sin quimioterapia, encontraron que la sobrevida media era significativamente mayor y la recurrencia se presentaba en menor porcentaje en los que recibieron quimioterapia coadyuvante. Detectaron ocho pacientes con cirugía incompleta (cirugía R1 o R2), entre los que no hubo diferencias de sobrevida entre los que recibieron y no recibieron quimioterapia. Con respecto a nueve pacientes, que recibieron quimioterapia inicial, existían sólo dos libres de enfermedad a los 12 y 20 meses de seguimiento. Existe un caso que recibió quimioterapia exclusiva, y que falleció siete años posteriores al tratamiento libre de enfermedad.

Aunque la resección tumoral logre ser completa, la recurrencia es común, es así como Lenze y colaboradores, detectaron en su revisión de casos que el $42 \%$ de los pacientes con cirugía R0 sin quimioterapia coadyuvante presentaban recurrencia, en promedio a los 8 meses de seguimiento, lo que concuerda con nuestro caso. Si bien la experiencia con la quimioterapia exclusiva o neoadyuvante es mínima en adultos, existen algunos casos con respuesta parcial y dos casos con respuesta completa, lo cual corroboraría que existirían tumores sensibles a la quimioterapia, y estos modelos de tratamiento podrían ser aplicables en casos seleccionados o con un alto riesgo quirúrgico.

Por último concluimos que se trata de un tumor de muy baja incidencia en la población adulta, por lo cual el dar a conocer las experiencias de los casos aislados en los distintos centros, es importante para poder mejorar las técnicas quirúrgicas, evaluar los beneficios de la quimioterapia, y proponer los esquemas más efectivos. El abordaje de esta patología requiere de un equipo con experiencia en métodos diagnósticos hepáticos y en resolución quirúrgica de ésta. Basados en la revisión de la experiencia publicada, la cirugía resectiva R0 sería el pilar fundamental del tratamiento, y la quimioterapia coadyuvante mejoraría los resultados tanto en disminuir la recurrencia como en aumentar la sobrevida.

\section{Referencias}

1. Lenze F, Birkfellner T, Lenz P, Hussein K, Länger F, Kreipe H, et al. Undifferentiated embryonal sarcoma of the liver in adults. Cancer 2008; 112: 2274-2282.

2. Weitz J, Klimstra DS, Cymes K, Jarnagin WR, 
D'Angelica M, La Quaglia MP, et al. Management of primary liver sarcomas. Cancer 2007; 109: 1391-1396.

3. Almogy G, Lieberman S, Gips M, Pappo O, Edden Y, Jurim $\mathrm{O}$, et al. Clinical outcomes of surgical resections for primary liver sarcoma in adults: results from a single centre. Eur J Surg Oncol 2004; 30: 421-427.

4. Jiménez Fuertes M, López Andújar R, de Juan Burgueño M, Moya Herráiz A, Sanjuán Rodríguez F, Montalvá Orón E, y cols. Sarcoma indiferenciado (embrionario) de hígado del adulto: informe de un caso y revisión de la literatura médica. Gastroenterol Hepatol 2008; 31: 12-17.

5. Buetow PC, Buck JL, Pantongrag-Brown L, Marshall WH, Ros PR, Levine MS, et al. Undifferentiated (embryonal) sarcoma of the liver: pathologic basis of imaging findings in 28 cases. Radiology 1997; 203: 779-783.

6. Dai CL, Xu F, Shu H, Xu YQ, Huang Y. Undifferentiated (embryonal) sarcoma of liver in adult: a case report. World J Gastroenterol 2005; 11: 926-929.

7. Avilés-Salas A, Garduño-Temblador JP, López-Basave H, Mondragón-Sánchez R. Sarcoma indiferenciado (embrionario) de hígado. Informe de un caso en una mujer adulta. Rev Gastroenterol Mex 2003; 68: 283-287.

8. Zheng JM, Tao X, Xu AM, Chen XF, Wu MC, Zhang SH. Primary and recurrent embryonal sarcoma of the liver: clinicopathological and immunohistochemical analysis. Histopathology 2007; 51: 195-203.

9. Ma L, Liu YP, Geng CZ, Tian ZH, Wu GX, Wang XL. Undifferentiated embryonal sarcoma of liver in an old female: case report and review of the literature. World $\mathrm{J}$ Gastroenterol 2008; 14: 7267-770.

10. Sakellaridis T, Panagiotou I, Georgantas T, Micros G, Rontogianni D, Antiochos C. Undifferentiated embryonal sarcoma of the liver mimicking acute appendicitis. Case report and review of the literature. World J Surg Oncol 2006; 20: 4-9.

11. Pachera S, Nishio H, Takahashi Y, Yokoyama Y, Oda K, Ebata T, et al: Undifferentiated embryonal sarcoma of the liver: case report and literature survey. J Hepatobiliary Pancreat Surg 2008; 15: 536-544.

12. Johnson JA 3rd, White JG, Thompson AR. Undifferentiated (embryonal) sarcoma of the liver in adults. Am Surg 1995; 61: 285-287.

13. Kadomatsu K, Nakagawara A, Zaizen Y, Nagoshi M, Tsuneyoshi M, Fukushige T, et al. Undifferentiated (embryonal) sarcoma of the liver: report of three cases. Surg Today 1992; 22: 451-455.

14. Tokunaga Y, Ryo J, Hoppou T, Kitaoka A, Tokuka A, Osumi K, et al. Hepatic undifferentiated (embryonal) sarcoma in an adult: a case report and review of the literature. Eur J Gastroenterol Hepatol 2000; 12: 12471251.

15. Grazi GL, Gallucci A, Masetti M, Jovine E, Fiorentino M, Mazziotti A, et al. Surgical therapy for undifferentiated (embryonal) sarcomas of the liver in adults. Am Surg 1996; 62: 901-906.

16. Kim M, Tireno B, Slanetz PJ. Undifferentiated embryonal sarcoma of the liver. AJR Am J Roentgenol 2008; 190: W261-262. 